\title{
Recidiva del cordoma sacrococcígeo. Colaboración multidisciplinaria
}

\author{
Recidiva of sacrococcygeal chordoma. Multidisciplinary collaboration
}

\author{
Ana Nogués-Pevidal'* ${ }^{*}$ Valentín Yuste-Benavente², Juan Casado Pellejero ${ }^{3}$, Juan Alberdi Viñas ${ }^{3}$ y \\ María Victoria Duque-Mallén ${ }^{1}$ \\ ${ }^{1}$ Servicio de Cirugía General y Aparato Digestivo, Sección de Cirugía Colorrectal; ${ }^{2}$ Servicio de Cirugía Plástica; ${ }^{3}$ Servicio de Neurocirugía. Hospital \\ Universitario Miguel Servet, Zaragoza, España
}

\section{Resumen}

Los cordomas son tumores derivados de los remanentes embrionarios de la notocorda. Son localmente invasivos y en estadios localizados la resección completa es el tratamiento de elección. Presentamos el caso de un varón de 77 años con recidiva de cordoma sacrococcígeo, intervenido por un grupo multidisciplinario formado por cirujanos coloproctólogos, plásticos y neurocirujanos, obteniendo resección en bloque y márgenes negativos. A pesar de que recidivan localmente un 66-75\% a los 5 años, la mayoría de los pacientes pueden aumentar su supervivencia si se obtienen márgenes libres. La cirugía debe conseguir una exéresis $R 0$ y minimizar el riesgo de lesión rectal.

Palabras clave: Cordoma sacrococcígeo. Recidiva. Tratamiento multidisciplinario.

\begin{abstract}
Chordomas are tumors arising from the embryonic remnants of the notochord. They are locally invasive and in localized stages, complete resection is the treatment of choice. We present the case of a 77-year-old man with recurrent sacrococcygeal chordoma, he was operated by a multidisciplinary group including colorectal-, plastic- and neurosurgeons, obtaining complete en bloc resection with tumor-free margins. Even though 66-75\% recur locally in 5 years, most of the patients can increase their survival if margins are tumor-free. The surgery must achieve the $R 0$ exeresis minimizing the risk of rectal injury.
\end{abstract}

Key words: Sacrococcygeal chordoma. Recurrence. Multidisciplinary treatment.

\section{Introducción}

Los cordomas son tumores primarios malignos derivados de los remanentes embrionarios de la notocorda, la cual desaparece en los seres humanos en la octava semana del desarrollo fetal; la evidencia sugiere que el cordoma se desarrolla a partir de elementos notocordales persistentes. Son inmunorreactivos para las citoqueratinas (CK) de bajo peso molecular, pero el antígeno de la membrana epitelial (EMA) y la expresión de S100 son variables ${ }^{1,2}$.

Corresponden al 1-4\% del total de los tumores óseos primarios y al $17.5 \%$ de los tumores óseos primarios malignos axiales. Tienen una incidencia anual

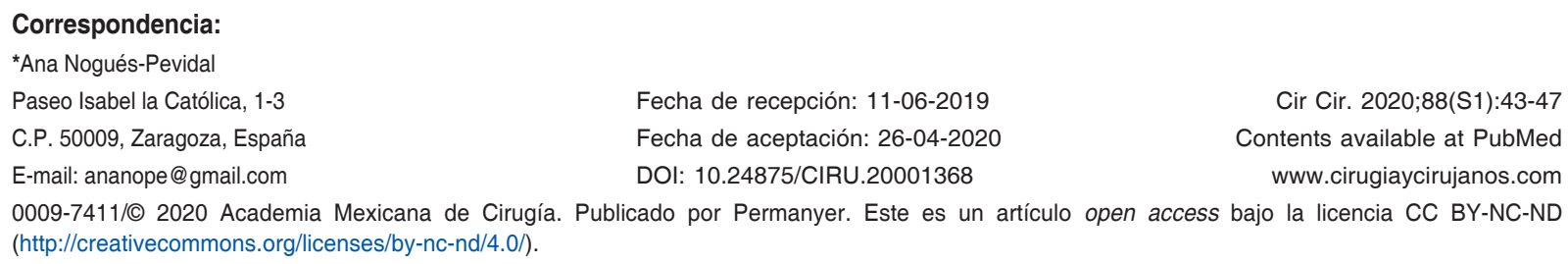

Fecha de recepción: 11-06-2019

Fecha de aceptación: 26-04-2020 DOI: $10.24875 /$ CIRU.20001368

Cir Cir. 2020;88(S1):43-47 Contents available at PubMed www.cirugiaycirujanos.com 0009-7411/@ 2020 Academia Mexicana de Cirugía. Publicado por Permanyer. Este es un artículo open access bajo la licencia CC BY-NC-ND (http://creativecommons.org/licenses/by-nc-nd/4.0/). 
de $0.08 / 10^{5}$ habitantes, y son más comunes en los hombres con una media de edad de 60 años. Su localización más frecuente es el sacro (50\%), seguida de la base del cráneo $(30 \%)$ y la columna vertebral $(20 \%)^{3}$.

Son neoplasias malignas localmente invasivas y de bajo grado (solo un $5 \%$ son agresivas). En estadios localizados, la resección quirúrgica completa con márgenes negativos es el tratamiento de elección. Tras la cirugía recidivan localmente un $66-75 \%$ a los 5 años, de los cuales un 30-40\% desarrollarán metástasis. En general, el progreso de la enfermedad depende más de su agresividad local que de su potencial metastásico².

Por este motivo, la recidiva del cordoma sacrococcígeo debe tener un enfoque multidisciplinario, en el cual el cirujano coloproctólogo desempeña un importante papel, dado que la técnica quirúrgica debe conseguir una exéresis R0 y minimizar el riesgo de lesión rectal.

\section{Presentación del caso}

Paciente varón de 77 años con antecedentes de hipertensión arterial, dislipidemia, síndrome de apnea obstructiva del sueño y estenosis aórtica, intervenido de artroplastia percutánea L4-L5 por estenosis lumbar.

En 2011 presenta una tumoración cutánea que se biopsia y se demuestra una neoplasia con rasgos morfológicos e inmunofenotípicos acordes con un cordoma, positivo para EMA, S100, CK19 y vimentina. Se realiza resonancia magnética (RM) de columna vertebral que confirma un cordoma sacrococcígeo de partes blandas que asocia infiltración anterior del músculo elevador del ano, en contacto con el recto, sin visualizar plano de clivaje.

Se decide realizar intervención quirúrgica por parte de los servicios de neurocirugía y de cirugía colorrectal. Se realiza una resección radical de la lesión que incluye coxis y $\mathrm{S} 5$, sin resección de recto porque no se observa infiltración. Sobre el mesorrecto expuesto se coloca una matriz de Duragen $®$, implante absorbible de colágeno utilizado como injerto superficial para reparación de defectos de la dura en intervenciones quirúrgicas craneales y de columna.

El informe de anatomía patológica confirma un cordoma que afecta la piel, las partes blandas y focalmente un fragmento de hueso, compatible con cordoma. La lesión contactaba con los extremos quirúrgicos. El estudio inmunohistoquímico (IHQ) es

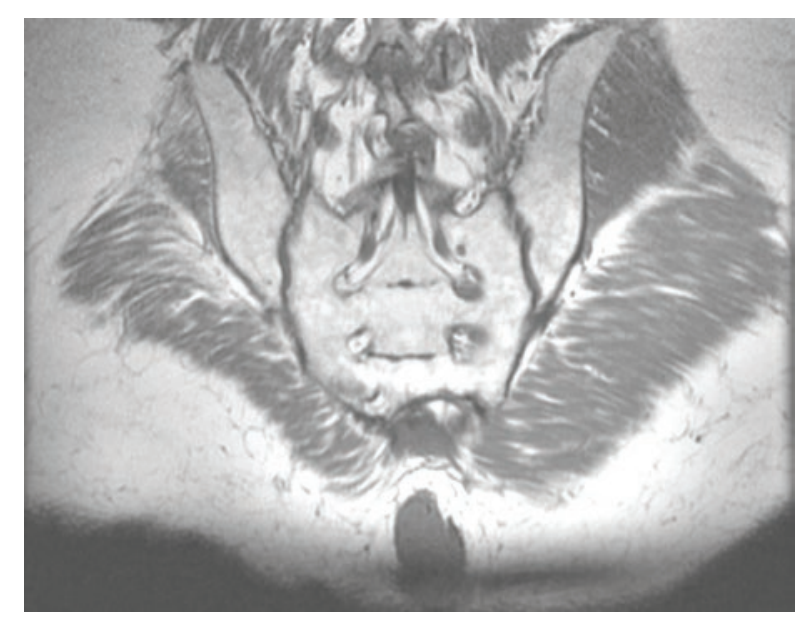

Figura 1. Lesión subcutánea interglútea.

positivo para EMA, S100, CK19 y vimentina, y negativo para CEA.

El seguimiento tras la intervención quirúrgica incluyó anamnesis, exploración física y RM de articulación sacroilíaca, semestral los primeros 3 años y anual posteriormente. El tercer año posoperatorio se identificó un resto tumoral en el lecho quirúrgico, que permaneció estable en las sucesivas revisiones, sin repercusión clínica, por lo que se optó por observación. El quinto año se diagnosticó como hallazgo casual una neoplasia de próstata, con extensión extracapsular y adenopatías regionales metastásicas. Tras ser confirmada con biopsia y descartada la enfermedad a distancia, se inició radioterapia pélvica con buena evolución.

El octavo año tras la cirugía se observa un aumento de tamaño de la tumoración en la región interglútea, de consistencia blanda (Fig. 1). En la RM sacra de control se identifica en el extremo inferior del sacro resecado, en la línea interglútea, una alteración subcutánea compatible con recidiva del cordoma. Los ejes principales son de $41 \times 17 \times 22 \mathrm{~mm}$ y presenta crecimiento tumoral respecto al estudio previo (Fig. 2). Según la octava clasificación TNM de tumores óseos, del American Joint Committee on Cancer (AJCC) del año 2019, la lesión corresponde a un estadio IA (rT1aNOM0) ${ }^{4}$.

La unidad de coloproctología planifica la intervención en colaboración con neurocirugía y cirugía plástica. Tras la preparación del colon y la administración de profilaxis antibiótica, se utiliza una vía posterior y se halla una lesión de $5 \mathrm{~cm}$ de localización subcutánea y en profundidad, con defecto óseo en la parte distal del hueso sacro y con la ampolla rectal que 


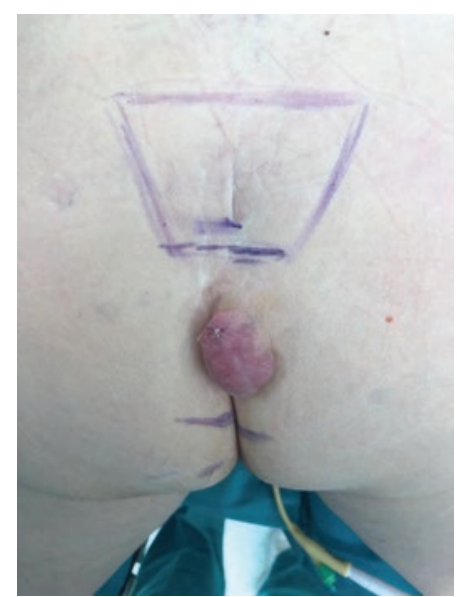

Figura 2. Resonancia magnética de sacro. En situación inferior al extremo resecado del sacro, en la línea interglútea, se observa una alteración subcutánea muy superficial, compatible con recidiva del cordoma. Los ejes principales miden $41 \times 17 \times 22 \mathrm{~mm}$.



Figura 3. Exéresis amplia. En la imagen de la izquierda se muestra el margen de piel alrededor de la lesión. En la imagen de la derecha se observa la sección de las inserciones del músculo glúteo.

protruye hacia el plano posterior al tacto rectal. Se realiza una exéresis completa de la lesión con márgenes de seguridad en la piel, el tejido subcutáneo, la fosa isquiorrectal y el espacio retrorrectal, guiada por tacto rectal dada la dificultad de identificar planos por la fibrosis causada por la cirugía y la colocación de malla previas (Figs. 3 y 4). El examen histológico intraoperatorio de los márgenes confirma su negatividad (Fig. 5) y se concluye la intervención con una plastia en $\mathrm{V}$ lateral a partir de la piel glútea izquierda (Fig. 6).

El posoperatorio cursa de forma favorable y el paciente es dado de alta el sexto día tras la intervención. Reingresa a las 2 semanas por infección de la herida, que se resuelve con curas y antibioticoterapia dirigida. La funcionalidad anorrectal no resultó comprometida.



Figura 4. Exéresis amplia (en profundidad, abomba la ampolla rectal).

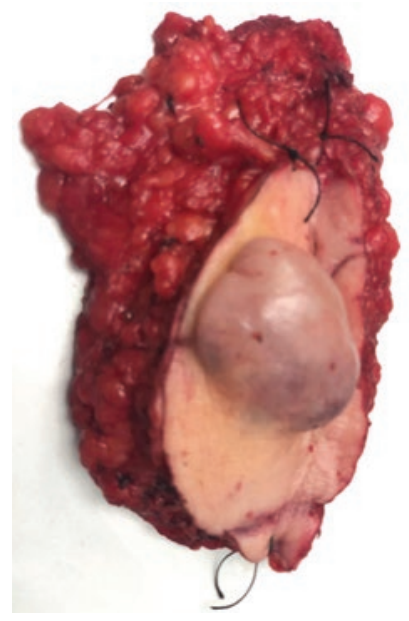

Figura 5. Pieza orientada.

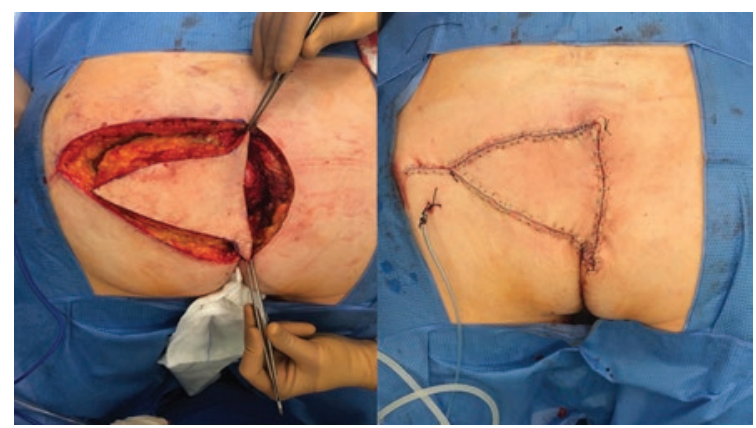

Figura 6. Plastia en V con piel del glúteo izquierdo.

El informe anatomopatológico describe una lesión de piel y de tejido subcutáneo compatible con cordoma, con márgenes quirúrgicos libres. El estudio IHQ 
resultó positivo para $\mathrm{CK}$ AE1-AE3 en las células tumorales. No se consideró necesario analizar otros marcadores.

\section{Discusión}

El cordoma es un tumor óseo maligno poco frecuente que ha tenido escasos tratamientos efectivos durante mucho tiempo. Actualmente hay disponibles nuevos tratamientos tanto para la fase local como para la fase avanzada de la enfermedad, pero existe incertidumbre ante la selección del más apropiado, lo que provoca variabilidad en su manejo en todo el mundo².

Ante la necesidad de un consenso global sobre el tratamiento de los pacientes con cordoma, en diciembre de 2013 la Sociedad Europea de Oncología Médica organizó una reunión con más de 40 expertos en varias disciplinas y la Chordoma Foundation, organización de defensa de los pacientes con cordoma, con el objetivo de elaborar un consenso sobre las pautas a seguir en su manejo y tratamiento. En noviembre de 2015, de nuevo organizaron una reunión con numerosos expertos para analizar los aspectos clave de la recurrencia locorregional del cordoma ${ }^{2-5}$.

Las opciones de tratamiento del cordoma incluyen cirugía, radioterapia y quimioterapia. La cirugía se asocia a una diferencia significativa en cuanto a supervivencia, en comparación con los pacientes que no son operados. La radioterapia se usa más frecuentemente en pacientes con recurrencia local y la quimioterapia está reservada para pacientes con metástasis ${ }^{6}$.

La resección quirúrgica completa con márgenes negativos en la enfermedad localizada es el método de referencia. Una resección R0 en bloque es el tratamiento recomendado cuando sea factible y el paciente acepte las secuelas; la esperanza libre de enfermedad a los 5 años en estos casos es superior al $50 \%$. La radioterapia adyuvante se recomienda en cordomas de la base del cráneo, la columna cervical y el sacro tras una resección R1. La radioterapia como único tratamiento se ofrece en determinados casos, cuando la resección no es posible o las secuelas no son aceptadas por el paciente. El cordoma tiene pocas opciones de tratamiento sistémico, con un beneficio limitado. La terapia dirigida podría ser un tratamiento alternativo razonable, pero los ensayos aleatorizados con un gran número de pacientes no son factibles en esta enfermedad rara? ${ }^{7}$.
Actualmente no hay un protocolo establecido de seguimiento, pero los expertos recomiendan realizar una RM del área del tumor primario semestral durante los primeros 4-5 años, y luego, si la enfermedad no progresa, anualmente durante 15 años. No hay consenso sobre la frecuencia ni el beneficio de la exploración del resto del cuerpo².

Respecto al tratamiento del cordoma primario sacrococcígeo, se recomienda la cirugía como primera opción terapéutica en los originados en S4 o en un nivel inferior. En los localizados en S3 la cirugía sigue siendo el principal tratamiento, especialmente si es posible preservar las raíces S2 porque, tras la cirugía, en un $40 \%$ de los casos se evidencia recuperación neurológica. Para los tumores originados por encima de S3, la cirugía siempre produce secuelas neurológicas importantes y la oportunidad de obtener un R0 es menor. En estos casos, la radioterapia es una alternativa en aquellos pacientes con sus funciones neurológicas intactas. La morbilidad asociada a la resección en bloque depende en gran medida del nivel de amputación sacra e incluye lesiones en el intestino, la vejiga y las raíces nerviosas, y su correspondiente deterioro funcional. Por este motivo, la planificación del tratamiento debe ser discutida y aceptada por un grupo multidisciplinario de especialistas, sopesando las secuelas y el deseo de obtener márgenes negativos ${ }^{2}$.

La recurrencia locorregional tiene una incidencia reportada del $66-75 \%$, la mayoría registradas en los primeros 5 años tras la cirugía. Los expertos recomiendan que la presencia o ausencia de síntomas debe tenerse en cuenta en la toma de decisiones, la confirmación histológica de la enfermedad recurrente no siempre es necesaria y las opciones de tratamiento de rescate con intención curativa incluyen cirugía y radioterapia, basándose en la evaluación de cada caso individual por un equipo multidisciplinario. En concreto, se puede considerar posponer el tratamiento de rescate en caso de enfermedad estable o ausencia de progresión de los síntomas ${ }^{5}$.

La recidiva del cordoma sacro ocurre principalmente en el hueso, aunque puede darse en otras localizaciones como el glúteo mayor o la fosa isquioanal. Se ha demostrado en estudios previos que tanto el nivel del tumor por encima de S3 como el margen quirúrgico son factores de riesgo significativos para la recidiva local. Dado que el margen quirúrgico es el único factor controlable en el tratamiento de la recurrencia, conseguir un margen libre es el objetivo más importante de las operaciones posteriores, 
obteniendo un beneficio clínico y aumentando la supervivencia en un gran número de pacientes ${ }^{8}$.

En conclusión, el caso clínico que presentamos muestra la necesidad de un consenso global sobre las pautas que hay que seguir en el manejo y el tratamiento de los pacientes con cordoma primario y recidivado, con el objetivo de mejorar los resultados y la supervivencia. Por otro lado, destaca el importante papel que desempeña el cirujano coloproctólogo en el tratamiento multidisciplinario de la recidiva del cordoma sacrococcígeo, dado que el objetivo es obtener una exéresis Ro minimizando el riesgo de lesión rectal. Desafortunadamente, obtener unos márgenes adecuados solo es posible en el $50 \%$ de los casos, lo que obliga en algunas ocasiones a incluir mesosigma o mesorrecto, e incluso a realizar una resección de recto y colostomía para obtener márgenes libres y evitar una infección ósea por una fístula colorrectal.

\section{Conflicto de intereses}

Los autores declaran no tener conflictos de intereses.

\section{Responsabilidades éticas}

Protección de personas y animales. Los autores declaran que para esta investigación no se han realizado experimentos en seres humanos ni en animales.

Confidencialidad de los datos. Los autores declaran que han seguido los protocolos de su centro de trabajo sobre la publicación de datos de pacientes.

Derecho a la privacidad y consentimiento informado. Los autores han obtenido el consentimiento informado de los pacientes y/o sujetos referidos en el artículo. Este documento obra en poder del autor de correspondencia.

\section{Bibliografía}

1. Zhang Z, Pang LJ, Wang N, Li Z, Cao YW, Hu WH, et al. Low-Grade Chondrosarcoma in the sellar area: Case report and literature review. Onco Targets Ther. 2019;12:10763-70.

2. Stacchiotti S, Sommer J; Chordoma Global Consensus Group. Building a global consensus approach to chordoma: a position paper from the medical and patient community. Lancet Oncol. 2015;16:e71-83.

3. D'Amore T, Boyce B, Mesfin A. Chordoma of the mobile spine and sacrum: clinical management and prognosis. J Spine Surg. 2018;4:546-52.

4. Gonzalez R. TNM staging of tumors of bone. PathologyOutlines.com website. Disponible en: http://www.pathologyoutlines.com/topic/bonestaging.html.

5. Stacchiotti S, Gronchi A, Fossati P, Akiyama T, Alapetite C, Baumann M, et al. Best practices for the management of local-regional recurrent chordoma: a position paper by the Chordoma Global Consensus Group. Ann Oncol. 2017;28:1230-42.

6. Yamada Y, Gounder M, Laufer I. Multidisciplinary management of recurrent chordomas. Curr Treat Options Oncol. 2013;14:442-53.

7. Alan O, Akin Telli T, Ercelep O, Tanrikulu Simsek E, Basoglu Tuylu T, Mutis A, et al. Chordoma: a case series and review of the literature. $J$ Med Case Rep. 2018;12:239.

8. Yang Y, Li Y, Liu W, Xu H, Niu X. The clinical outcome of recurrent sacral chordoma with further surgical treatment. Medicine (Baltimore). 2018;97:e13730. 